\title{
VALVULAR DISEASE
}

\section{Improving outcomes for aortic valve replacement in the USA}

For individuals with aortic valve disease, replacement of the defective valve is the only theraputic option to prolong life. Most patients receive a prosthetic valve fitted by a surgeon, but new transcatheter techniques are now approved for high-risk patients and individuals with inoperable disease. Two papers, both published in $J A M A$, describe the outcomes for patients undergoing aortic valve replacement (AVR) in the USA by either transcatheter or surgical-based methods.

In a large cross-sectional cohort study, Barreto-Filho and colleagues analysed the outcomes of $>82$ million patients who

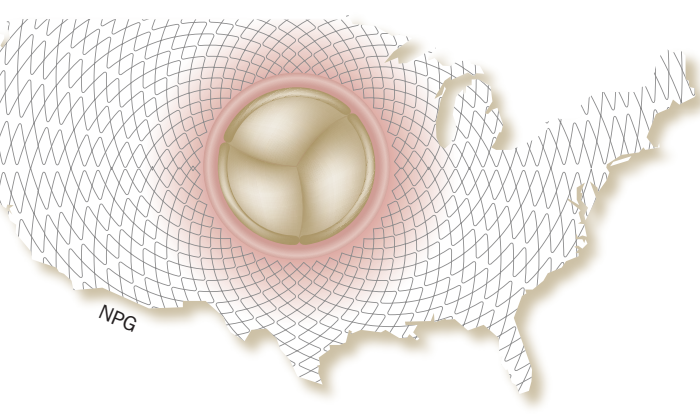

underwent AVR surgery between 1999 and 2011 in the US Medicare system. The overall rate of surgical AVR intervention increased over the 12-year period, but the clinical outcomes improved. Absolute 30-day and 1-year mortality dropped by $3.4 \%$ (95\% CI $3.0-3.8 \%)$ and $2.6 \%$ (95\% CI 2.1-3.2), respectively. However, these improved outcomes were not universal across all demographics. Female and African-American patients both underwent fewer procedures and had worse outcomes compared with the rest of the population. Interestingly, despite new advances in prosthetic valves, in 2011 nearly $24 \%$ of patients aged $>85$ years still received a mechanical device.

Surgery is not appropriate for all patients, and in 2011 the FDA approved the use of transcatheter-based aortic valve replacement (TAVR) in patients with inoperable valve disease. This approval was extended in 2012 to patients who have operable disease, but are deemed to be high-risk surgical candidates. Mack and colleagues have reported the outcomes from 224 hospitals contributing to the TVT Registry of TAVR outcomes.

Between November 2011 and May 2013, 7,710 TAVR procedures were performed, and $92 \%$ of these procedures were successful. In-hospital mortality among patients who underwent successful TAVR procedures was 5.5\% (95\% CI 5.0$6.1 \%)$. Among patients for whom 30 -day follow-up data was available $(n=3,133)$, mortality was 7.6\% (95\% CI 6.7-8.6\%), but over half of the deaths were attributed to a noncardiovascular event. The results are promising, and comparable with those of various European registries, but the authors recognise the need for a longerterm follow-up to assess ongoing safety and effectivness.

Tim Geach

Original articles Barreto-Filho, J. A. et al. Trends in aortic valve replacement for elderly patients in the United States, 1999-2011. JAMA 310, 2078-2085 (2013) | Mack, M. J. et al. Outcomes following transcatheter aortic valve replacement in the United States. JAMA 310, 2069-2077 (2013) 